\title{
Quiz Questions on Anemia
}

Anemia, the commonest blood disorder still poorly understood, is prevalent from newborn, to adolescents, in pregnancy, and till menopause! A small quiz may give some answers.

Q1. Which of the following is NOT a cause of microcytic anemia?
a. Thalassemia
b. Anemia of chronic disease
c. Iron deficiency anemia
d. Pancytopenia
e. Lead poisoning

Q2. The lab reports for a patient with low mean cell volume show high serum ferritin and low total iron binding capacity. What is the most likely cause for this patient's anemia?
a. Fe deficiency
b. Anemia secondary to inflammation
c. Thalassemia
d. Hemoglobinopathy

Q3. Fe is absorbed in the
a. Stomach
b. Duodenum
c. Jejunum
d. Ileum

Q4. Where is most nonheme iron found in the body?
a. Bound to IF
b. Bound to transferrin
c. Free in plasma
d. Stored in liver

Q5. Select the following that enhance Fe absorption (select all that apply)
a. Citric acid
b. Polyphenols (tea)
c. Phytate (bran)
d. Calcium
e. Ascorbic acid

Q6. What is the most important test for Fe stores?
a. Serum iron
b. TIBC
c. Serum ferritin

Q7. Which of the following is not an etiology of Fe deficiency anemia?
a. Chronic blood loss
b. Increased requirement
c. Infection
d. Malabsorption
e. Decreased intake

Q8. TIBC increases in iron deficiency anemia because
a. Inflammatory response to deficiency
b. Compensation by other factors
c. Ability to absorb increases

Q9. Pica, a clinical presentation for Fe deficiency anemia, is
a. Itchiness
b. ED
c. Desire to eat weird things
d. A small woodland creature

Q10. Which lab investigations would you order if you suspect Fe deficiency anemia? (check all that apply)
a. $\mathrm{CBC}$
b. Blood smear
c. Serum iron
d. Serum ferritin
e. TIBC
f. All of the above

Q11. Where is beta-thalassemia most common? (check all that apply)
a. West Africa
b. Mediterranean
c. Arabian Peninsula
d. South East Asia
e. Canada

Q12. What is the difference between beta-thalassemia major and beta-thalassemia minor?
a. Homozygous vs heterozygous
b. Acute vs chronic
c. Legal drinking age

Q13. Heinz bodies are made of:

a. Excess gamma chains

b. Excess alpha chains

c. Excess beta chains

d. Excess ketchup 
Q14. Beta-thalassemia, unlike alpha-thalassemia, presents at approximately 6 months of age.
a. True
b. False

Q15. Which would you expect to see on a blood smear for beta-thalassemia? (select all that apply)
a. Heinz bodies
b. Multinucleated neutrophils
c. Target cells
d. Hypochromic microcytic cells
e. Hyperchromic microcytic cells

Q16. What is the treatment for beta-thalassemia minor?
a. Blood transfusions
b. Iron chelation
c. Bone marrow transplant
d. None of the above

Q17. Decreased or stopped production of alpha-globin chains results in $\mathrm{HbH}$ (4 gamma chains together) and $\mathrm{Hb}$ Barts ( 4 beta chains together)

a. True

b. False

Q18. On a CBC for alpha-thalassemia, you would see anemia and reticulocytosis. On the blood smear, you would see Heinz bodies, hypochromic microcytic cells, and occasional target cells. Select the others that you would see increase:
a. $\mathrm{LDH}$
b. Unconjugated bilirubin
c. Conjugated bilirubin
d. Urine urobilinogen
e. Urine hemosiderin

Q19. Aplastic anemia can be acquired (more common) and inherited. What are some of the ways it can be acquired?
a. Postviral infection
b. Pregnancy
c. Ionizing radiation
d. Drugs and chemicals
e. Idiopathic
f. All of above

Q20. Aside from the gradual onset signs of anemia, what other clinical presentations would you see with aplastic anemia?
a. Koilonychias, "spoon nails"
b. Associated thrombocytopenia, e.g., history of bleeding from the gums
c. Neutropenia, e.g., repeated bacterial infections
d. Purpura
e. Pica

Q21. How would you diagnose aplastic anemia?
a. Blood smear
b. Bone marrow biopsy
c. Spleen biopsy
d. $\mathrm{CBC}$
e. Liver biopsy

Q22. Select treatment options for aplastic anemia
a. IV equine ATG
b. Bone marrow transplant
c. Splenectomy
d. Immune suppression 